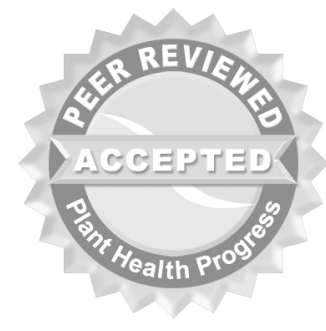

(c) 2010 Plant Management Network.

Accepted for publication 18 November 2010. Published 27 December 2010.

\title{
Seed Transmissibility of Alfalfa mosaic virus in Soybean
}

\author{
B. He, O. L. Fajolu, R.-H. Wen, and M. R. Hajimorad, Department \\ of Entomology and Plant Pathology, The University of Tennessee, \\ Knoxville, TN 37996
}

Corresponding author: M. R. Hajimorad. mrh@utk.edu

He, B. Fajolu, O. L., Wen, R.-H., and Hajimorad, M. R. 2010. Seed transmissibility of Alfalfa mosaic virus in soybean. Online. Plant Health Progress doi: 10.1094/PHP-20101227-01-BR.

Alfalfa mosaic virus (AMV; genus Alfamovirus; family Bromoviridae) is considered an emerging viral disease of soybean [Glycine max (L.) Merrill] in the midwestern United States. This is partly attributed to the introduction of the soybean aphid (Aphis glycines) (4). In Wisconsin, AMV was associated with soybean yield reductions of 31 and $26 \%$ in 2002 and 2003, respectively (4). AMV seed transmissibility has been well demonstrated in a number of crops such as alfalfa (2). However, in regard to soybean, statements on AMV seed transmission have been published without supportive experimental evidence, and in one instance detected based on symptomatology in a single cultivar (1).

To provide unequivocal experimental evidence, we mechanically inoculated soybean cultivars Colfax, Lee68, and Williams82 with sap from soybean leaves infected with strains $\mathrm{H}_{4}$, N2O, S30, S4O, and W1 from lucerne (ATCC type strains PV845 to 849, respectively). AMV strains Joe Davis and Champaign from soybean (3) were also mechanically inoculated to Williams82. Each of the strains was singly inoculated to four replicate plants of each cultivar and maintained in a growth chamber at $25^{\circ} \mathrm{C}$ with a photoperiod of $16 \mathrm{~h}$. Infection was confirmed by ELISA (3). The infected plants exhibited a wide range of systemic symptoms while grown to maturity. Strains N20, S30, and W1 infected all the cultivars whereas $\mathrm{H}_{4}$ and $\mathrm{S} 40$ failed to infect Colfax. AMV-N2O infection in Lee68 and Williams82 was lethal. AMV strains Joe Davis and Champaign infected Williams82.

Mature seeds were harvested; those from the same cultivar infected with the same strain were combined and stored at room temperature. Seeds did not exhibit mottling; however, those from the same cultivar infected with different strains exhibited size and color differences (Fig. 1). Seeds were grown under similar conditions as the parental plants. Some of the seedlings exhibited typical symptoms of AMV infection, while others remained symptomless (Fig. 2). A leaflet from each leaf of each seedling was harvested at 28 days after planting and combined. Each seedling was evaluated singly by ELISA. AMV-S4O was not seed transmissible in any of the cultivars. AMV-H4 was seed transmissible in Lee68 and Williams82 at rates of 9 and 4\%, respectively. AMV-S30 was not seed transmissible in Lee68 or Williams82, but transmitted at a rate of $8 \%$ in Colfax. AMV-N2O was not seed-transmissible in Colfax. AMV-W1 was seed transmissible in Lee68 at a rate of $5 \%$, but not in Colfax or Williams82. AMVChampaign was not seed transmissible in Williams82, but AMV-Joe Davis transmitted at a rate of $9 \%$. These variations are not surprising as seed transmissibility is virus strain and host-genotype dependent (2). 


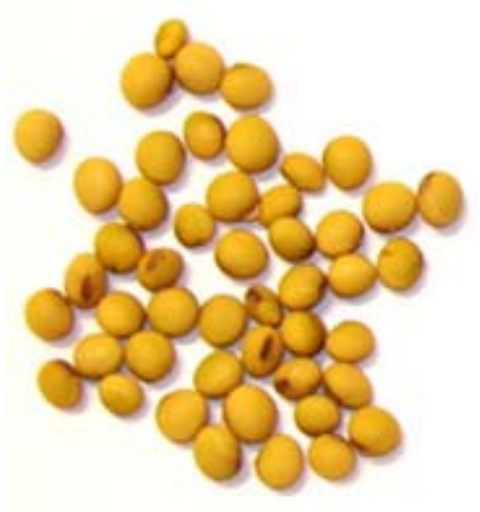

AMV-W1

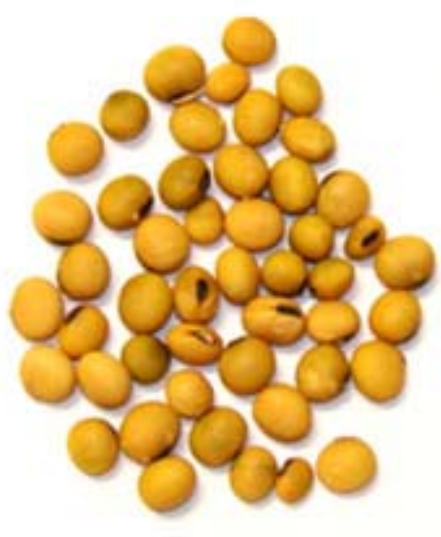

AMV-N20

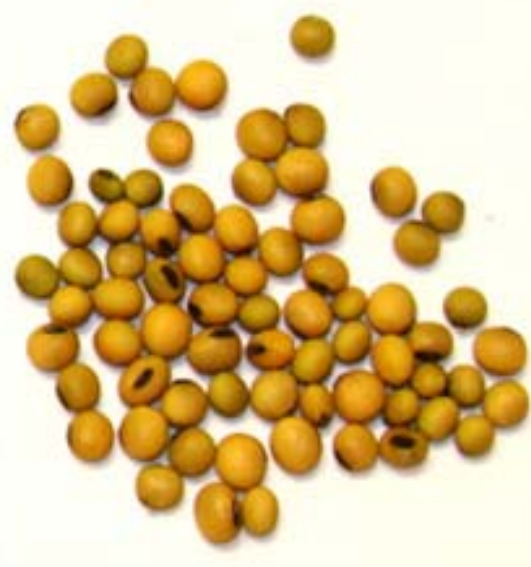

AMV-S30

Fig. 1. Phenotype of seeds derived from soybean cv. Colfax infected with three AMV strains.

\section{AMV-Joe Davis}

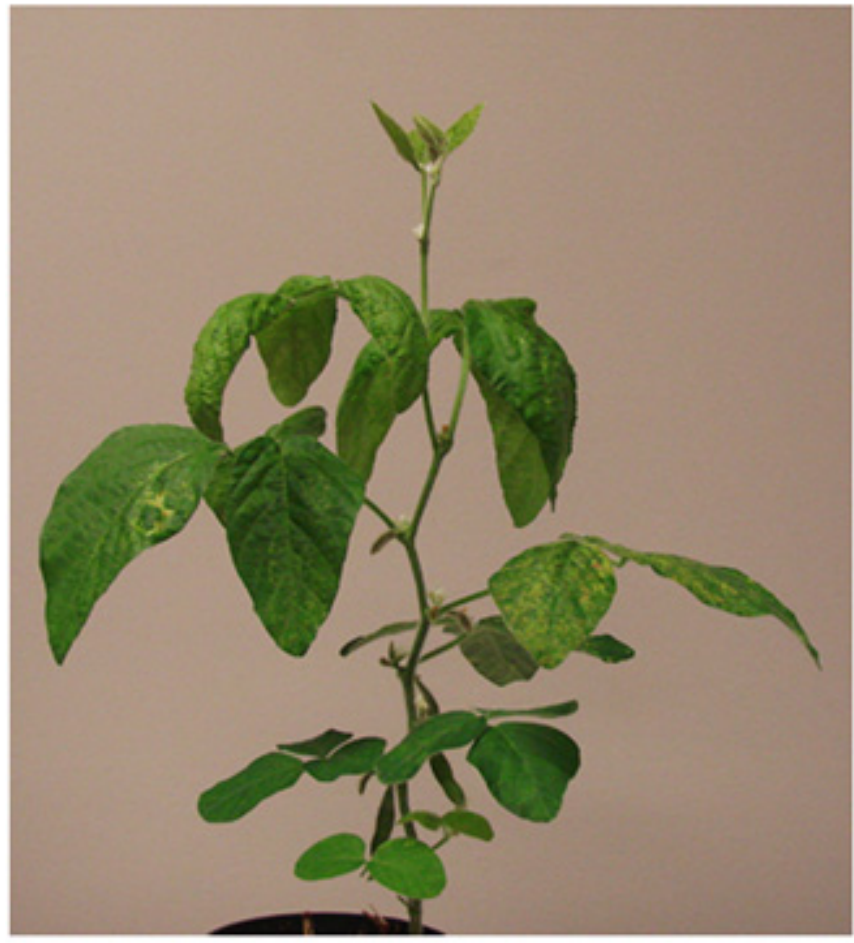

\section{Williams82}

AMV-S30

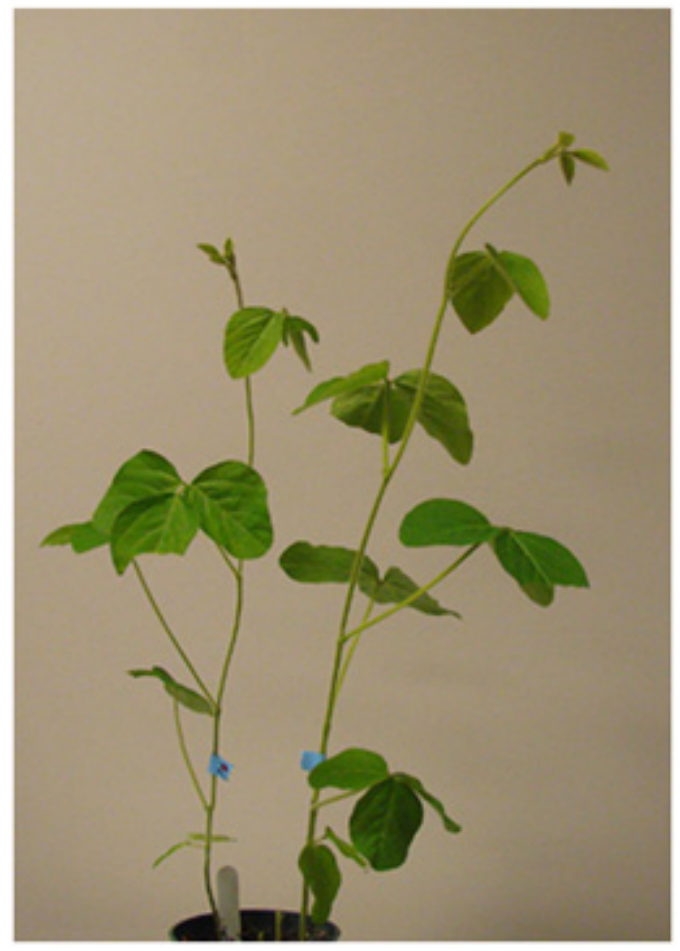

Colfax

Fig. 2. Phenotypes of two AMV strains following seed transmission. Photographs of the plants were taken 75 (Williams82) and 35 (Colfax) days past planting. Note that AMV-S30 induced symptoms, unlike that of AMV-J oe Davis, are not pronounced. 
We validated our finding by RT-PCR where total RNA was extracted from two AMV-S30 infected Colfax seedlings and served as template for cDNA synthesis using random primers. The cDNAs were subsequently used as templates in PCR in the presence of primers 1193 (forward) (5'-

AGCTGAATTCATGAGTTCTTCACAAC-' 3 ) and 1858 (reverse) (5'GCTAGCGGCCGCTCAATGACGATC-3') corresponding to sequences 1193 to 1210 and 1858 to 1840 of RNA3 from AMV-Kr (GenBank Accession No.

$\mathrm{AB} 126032)$, respectively. The purified amplicons were directly sequenced using the same primers. Sequences derived from the two seedlings were identical and Blast analysis revealed $100 \%$ identity with the coat protein sequences of AMVS30 (HM807305).

Collectively, this report provides unequivocal evidence on AMV seed transmission in soybean. Infected seed may serve as a reservoir for survival and spread of AMV in soybean.

\section{Literature Cited}

1. Iizuka, N., and Yoshida, K. 1988. Incidence of mosaic disease in soybean in Hokkaido, and seed transmission of the causal viruses. Res. Bull. Hokkaido Nat. Agric. Exp. Stn. 150:33-43.

2. Johnsen, E., Edwards, M. C., and Hampton, R. O. 1994. Seed transmission of viruses-current perspectives. Annu. Rev. Phytopath 32:363-386.

3. Malapi-Nelson, M., Wen, R.-H., Ownley, B. H., and Hajimorad, M. R. 2009. Coinfection of Soybean mosaic virus and Alfalfa mosaic virus results in disease synergism and alteration in accumulation level of both viruses. Plant Dis. 93:12591264.

4. Mueller, E. E., and Grau, C. R. 2007. Seasonal progression, symptom development, and yield effects of Alfalfa mosaic virus epidemics on soybean in Wisconsin. Plant Dis. 91:266-272. 